\title{
Doença de Kawasaki e COVID-19: uma revisão de literatura
}

\author{
Kawasaki and COVID-19 disease: a literature review \\ Enfermedad de Kawasaki y COVID-19: revisión de la literatura
}

Débora Karine dos Santos Pacífico ${ }^{1 *}$, Francisco David de Souza e Silva ${ }^{1}$, Antonione Santos Bezerra Pinto $^{1}$, Edilberto Ferreira Veras Junior ${ }^{1}$, Davi Sérgio dos Santos Pacífico ${ }^{1}$, Vinícius José de Melo Sousa $^{1}$, João Henrique Piauilino Rosal ${ }^{1}$, Larissa Oliveira Batista ${ }^{2}$.

\section{RESUMO}

Objetivo: Identificar a relação entre a Doença de Kawasaki e o novo Coronavírus (COVID-19). Revisão bibliográfica: Foram analisados 91 artigos, sendo 39 lidos integralmente e utilizados neste estudo. A análise viabilizou a percepção de que infecções pelo novo Coronavírus levam a uma resposta hiperinflamatória ou tempestade de citocinas, como interleucinas (IL) 1 e 6 , ativação de macrófagos e presença de imunoglobulina (Ig) A, provocando patologias como linfohistiocitose hemofagocítica secundária, Síndrome de Disfunção Respiratória Aguda (SDRA) e alterações nas artérias coronarianas semelhantes à Doença de Kawasaki. O dano epitelial provocado pelo vírus em estágio inicial, assim como na DK, pode induzir à endotelite local secundária, o que pode explicar um fenótipo vasculítico autoinflamatório tardio com regulação positiva de interleucinas. Essa inflamação endotelial aumentada e lesão/disfunção após a infecção pelo novo Coronavírus, provavelmente, é devido à ação da enzima conversora de angiotensina (ECA). Considerações finais: Nos últimos 20 anos, os vírus da família dos Coronavírus foram propostos como participantes na patogênese da doença de Kawasaki (DK), uma vasculite sistêmica aguda. A associação documentada levanta a preocupação de saber se a infecção por COVID-19 aumenta o risco de DK.

Palavras-chave: COVID-19, Citocinas, Doença de Kawasaki.

\begin{abstract}
Objective: To identify the relationship between Kawasaki disease and the new Coronavirus (COVID-19). Bibliographic review: 91 articles were analyzed, 39 of which were read in full and used in this study. The analysis made possible the perception that infections by the new Coronavirus lead to a hyperinflammatory response or cytokine storm, such as interleukins (IL) 1 and 6, activation of macrophages and the presence of immunoglobulin (lg) A, causing pathologies such as secondary hemophagocytic lymphohistocytosis, Syndrome of acute respiratory dysfunction and changes in coronary arteries similar to Kawasaki disease. Epithelial damage caused by the virus at an early stage, as well as in KD, can induce secondary local endothelitis, which may explain a late auto-inflammatory vasculitic phenotype with positive regulation of interleukins. This increased endothelial inflammation and injury / dysfunction after infection with the new Coronavirus is probably due to the action of the angiotensin-converting enzyme (ACE). Final considerations: In the last 20 years, viruses of the Coronavirus family have been proposed as participants in the pathogenesis of Kawasaki disease (DK), an acute systemic vasculitis. The documented association raises concerns about whether COVID-19 infection increases the risk of KD.
\end{abstract}

Keywords: COVID-19, Cytokines, Kawasaki disease.

\footnotetext{
${ }^{1}$ Instituto de Educação Superior do Vale do Parnaíba (IESVAP), Parnaíba - PI.

*E-mail: deborakrine02@gmail.com

2 Universidade Estadual do Piauí (UESPI), Teresina - PI.
} 


\section{RESUMEN}

Objetivo: Identificar la relación entre la enfermedad de Kawasaki y el nuevo Coronavirus (COVID-19). Revisión bibliográfica: Se analizaron 91 artículos, de los cuales 39 fueron leídos íntegramente y utilizados en este estudio. El análisis posibilitó la percepción de que las infecciones por el nuevo Coronavirus conducen a una respuesta hiperinflamatoria o tormenta de citocinas, como las interleucinas (IL) 1 y 6 , la activación de macrófagos y la presencia de inmunoglobulina (lg) A, provocando patologías como la linfohistocitosis hemofagocítica secundaria, Síndrome de disfunción respiratoria aguda y cambios en las arterias coronarias similares a la enfermedad de Kawasaki. El daño epitelial causado por el virus en una etapa temprana, así como en la EK, puede inducir endotelitis local secundaria, lo que puede explicar un fenotipo vasculítico autoinflamatorio tardío con regulación positiva de interleucinas. Este aumento de la inflamación y la lesión / disfunción endotelial después de la infección con el nuevo coronavirus probablemente se deba a la acción de la enzima convertidora de angiotensina (ECA). Consideraciones finales: En los últimos 20 años, los virus de la familia Coronavirus se han propuesto como participantes en la patogenia de la enfermedad de Kawasaki (DK), una vasculitis sistémica aguda. La asociación documentada genera preocupaciones sobre si la infección por COVID-19 aumenta el riesgo de EK.

Palabras clave: COVID-19, Citoquinas, Enfermedad de Kawasaki.

\section{INTRODUÇÃO}

A COVID-19 causada por SARS-CoV-2, foi declarada uma pandemia global pela Organização Mundial da Saúde (OMS) em 11 de março de 2020, sendo que os sintomas mais comumente relatados incluem tosse, febre e falta de ar (WALTUCH T, et al., 2020).

Esse vírus acessa as células hospedeiras através da enzima conversora de angiotensina 2 (ECA2), que é abundante nos pulmões e nas células endoteliais (SARDU C, et al., 2020). Pode-se destacar uma ligação entre as formas graves da contaminação e a chamada tempestade de citocinas, também com níveis aumentados de ferritina (ALUNNO A, et al., 2020).

Nas crianças, o envolvimento respiratório parece ter um curso mais benigno, com quase nenhuma fatalidade relatada, já que apresentam imunidade cruzada de outras infecções virais e/ou o sistema imunológico em desenvolvimento pode responder ao novo Coronavírus de maneira diferente. Porém, o trato respiratório não é o único sistema suscetível à infecção pelo SARS-CoV-2, pois evidências recentes sugerem que o dano tecidual é principalmente mediado pela imunidade inata do hospedeiro. Esta infecção é caracterizada por uma tempestade de citocinas semelhante à ativação de macrófagos, observada na linfohistiocitose hemofagocítica (HLH) induzida por vírus (VERDONI L, et al., 2020).

O Sistema Nacional de Saúde (SNS) do Reino Unido e a Sociedade de Terapia Intensiva Pediátrica (STIP) emitiram um alerta recentemente sobre a ocorrência de cerca de 20 casos da chamada Síndrome Inflamatória Multissistêmica Pediátrica (MIS-C), associada temporariamente ao novo Coronavírus (RAUF A, et al., 2020). Desde o início de abril, os casos de choque hiperinflamatório pediátrico continuam a aumentar (DEY M e ZHAO SS, 2020).

Houve casos na idade pediátrica caracterizados por choque, miocardite e sinais variáveis de inflamação multissistêmica, que ocorreram com mais frequência em algumas áreas, como na Europa e no Leste do EUA (KONÉ-PAUT I e CIMAZ R, 2020). Essa síndrome inflamatória multissistêmica compartilha características sobrepostas com outras condições inflamatórias pediátricas, como a Doença de Kawasaki (DK) e a Síndrome de Choque Tóxico (SCT) (RAUF A, et al., 2020).

A Doença de Kawasaki (DK) é uma doença febril sistêmica aguda que afeta principalmente crianças com menos de 5 anos de idade. Tosse, vômito, piúria estéril e diarreia são muito comuns (ALIZARGAR J, 2020). A doença é mais comum no sudeste da Ásia, incluindo crianças asiáticas que vivem em outros lugares, sugerindo uma predisposição genética. A infecção pode desempenhar um papel, principalmente devido à prevalência sazonal de casos que atingem o pico durante a primavera-inverno (DEY M e ZHAO SS, 2020). 
A etiologia exata da DK não está totalmente elucidada, mas doenças infecciosas, principalmente as causadas pelos vírus respiratórios, como Enterovírus, Adenovírus, Rinovírus e Coronavírus, têm sido relatadas como fatores predisponentes (ALIZARGAR J, 2020).

Nos estágios iniciais da DK, pode-se observar um influxo de neutrófilos, com uma rápida transição para grandes células mononucleares em conjunto com linfócitos e células plasmáticas de imunoglobulina A (IgA). Destruição da lâmina elástica interna e eventual proliferação de fibroblastos podem ocorrer de forma tardia. A inflamação ativa é substituída, em várias semanas a meses, por fibrose progressiva, com formação e remodelação de cicatrizes (RIGANTE D, 2020).

Devido ao número muito maior de infecções e à facilidade de transmissão, o novo Coronavírus pode representar um risco maior para doença de Kawasaki ou sintomas semelhantes a Kawasaki (PRUC M, et al., 2020). Diante deste cenário atual pandêmico, esta revisão apresenta as evidências científicas disponíveis, até o momento, sobre a associação da Doença de Kawasaki e o novo Coronavírus.

\section{REVISÃO BIBLIOGRÁFICA}

\section{Características epidemiológicas e fisiopatológicas da Doença de Kawasaki:}

A doença de Kawasaki (DK) é uma vasculite sistêmica da infância que pode afetar as artérias coronárias (DURSUN R e TEMIZ SA, 2020). Apresenta-se como uma condição inflamatória sazonal e rara, mas potencialmente grave em crianças, ocorrendo principalmente em menores de cinco anos (XU S, et al., 2020).

Algumas evidências sugerem que existem múltiplos fatores que desempenham papeis diferentes na patogênese da DK, como predisposição genética, autoimunidade, etiologia infecciosa e resposta imune inflamatória do hospedeiro (RABA AA e ABOBAKER A, 2020).

Uma forma grave de complicação é o aneurisma da artéria coronária (AAC), cuja ruptura leva à formação de trombose e infarto do miocárdio (XU S, et al., 2020). A interleucina (IL) 1, em particular, tem sido implicada na formação de AAC. Esse acometimento ocorre dentro de 2 semanas, durante a fase convalescente do DK, sendo tipicamente identificado por ecocardiografia (LOKE YH, et al., 2020).

Essa é uma doença mundial, com taxas de incidência variáveis que refletem principalmente a composição racial das populações de vários países. A maior incidência de DK é no Japão, e isso tem aumentado constantemente com uma taxa anual de 0,308\% em crianças menores de 5 anos relatadas em 2014.

No Japão, uma em cada 65 crianças desenvolve DK aos 5 anos. Mais de 15.979 casos de DK foram relatados no Japão em 2015 com aglomerados locais ocorrendo comumente. A segunda maior taxa relatada foi $0,1997 \%$ em menores de 5 anos de idade em 2014 na Coreia do Sul, enquanto Taiwan teve a terceira maior taxa (0,0828\%) em menores de 5 anos em 2010 (ROWLEY AH e SHULMAN ST, 2018).

As diferenças muito marcantes nas taxas étnicas específicas são indicativas de uma base genética de suscetibilidade muito forte. A proporção de pacientes com DK do sexo masculino/sexo feminino é de aproximadamente 1,5: 1 em virtualmente todos os países, e as complicações cardíacas graves de DK são ainda mais significativamente representados em homens, sendo que a base da preponderância masculina não é clara.

A doença de Kawasaki (DK) tem uma distribuição de idade marcante, com quase $100 \%$ dos casos ocorrendo em crianças, $80 \%$ em crianças menores de 5 anos e $50 \%$ em menores de 2 anos (ROWLEY AH e SHULMAN ST, 2018).

Suscetibilidade genética é uma das áreas de investigação em andamento em DK, dada a especificidade padrões de ocorrência desta condição em diferentes grupos étnicos e raças. Os estudos iniciais sobre a base genética de DK foram focados no Antígeno Leucocitário Humano (HLA) e descobriram que HLA-DRB1, HLA B5, Bw51 e Bw44 estão associados à susceptibilidade a DK.

Alguns dos genes mais amplamente estudados a serem encontrados consistentemente associados com DK incluem inositol 1,4,5-trifosfato 3-quinase (ITPKC), caspase-3 (CASP3), linfócito B quinase (BLK), CD40 e HLA (AGARWAL S e AGRAWAL DK, 2017). 
Acredita-se que a DK ocorra como consequência de um sistema imunológico desregulado. As células T foram implicadas na imunopatogênese da DK com envolvimento do ITPKC, o qual funciona como mediador principal. O ITPKC é um regulador negativo da ativação das células $T$ e a perda de seu controle leva ao aumento da ativação das células $T$ e produção de citocinas, que foi demonstrada em pacientes com DK. Recentemente, estudos mostraram um desequilíbrio de células Th (células T helper) e células Treg (células T regulatórias) em DK aguda (AGARWAL S e AGRAWAL DK, 2017).

A patologia das alterações da artéria coronária envolve três fases: arterite necrosante na fase aguda, com neutrófilos destruindo a parede (túnica media à túnica adventícia) levando à formação aneurismática; Vasculite subaguda ou crônica envolvendo linfócitos $T$, células $B$ plasmáticas e macrófagos infiltrando a parede do vaso; e proliferação miofibroblástica luminal, envolvendo miofibroblastos e deposição de matriz ao longo de meses e anos contribui para a estenose arterial (LOKE YH, et al., 2020).

Logo, essa doença causa inflamação vascular e restringe o fluxo sanguíneo ao coração (XU S, et al., 2020). No que diz respeito à patogênese da DK, ainda não está clara, mas é o resultado de uma resposta imune excessiva a agentes desencadeantes não identificados, o que poderia estimular o sistema imunológico inato com forte resposta inflamatória sistêmica e envolver imunidade adaptativa, causando danos aos órgãos (KONÉ-PAUT I e CIMAZ R, 2020).

Os pacientes com apresentações do tipo Kawasaki foram definidos de acordo com os critérios da American Heart Association, incluindo o tipo clássico (febre por $\geq 5$ dias mais quatro ou mais critérios clínicos, incluindo conjuntivite não exsudativa bulbar bilateral, alterações dos lábios ou cavidade oral, linfadenopatia laterocervical não supurativa, erupção cutânea polimórfica, eritema das palmas das mãos e plantas dos pés, endurecimento firme das mãos ou pés, ou ambos) e os tipos incompletos. Nestes (febre por $\geq 5$ dias mais dois ou três dos critérios clínicos mencionados), os valores da taxa de sedimentação de eritrócitos (VHS) e/ou proteína $C$ reativa $(P C R)$ foram considerados como um critério diagnóstico adicional em associação com presença de anemia, trombocitose após 7 dias de febre, hipoalbuminemia, hipertransaminemia, leucocitose, piúria estéril ou um ecocardiograma mostrando aneurismas coronarianos ou disfunção cardíaca (ou seja, depressão da função ventricular esquerda, insuficiência valvar mitral ou derrame pericárdico) (VERDONI L, et al., 2020).

Os biomarcadores cardíacos são utilizados para fins de diagnóstico, mas o monitoramento regular por meio de exames de imagem é primordial, sendo preconizadas as técnicas como imagem de perfusão do miocárdio, angiografia e mais recentemente a ressonância magnética (GONÇALVES LF, et al., 2020). Pacientes em fase aguda de DK apresentam tipicamente leucocitose com predominância de neutrófilos.

O fragmento N-terminal do peptídeo natriurético do tipo B (NT-proBNP) é comumente elevado em pacientes com DK e é útil no diagnóstico com sensibilidade combinada de 80 - 89\% (LOKE YH, et al., 2020). Os pacientes devem receber terapias que foram usadas com sucesso no tratamento de Doença de Kawasaki, incluindo imunoglobulina intravenosa (IgIV) e metilprednisolona. Na maioria dos pacientes, isso foi altamente eficaz na redução da inflamação sistêmica, como evidenciado pela resolução da febre (CHIOTOS K, et al., 2020).

A American Academy of Pediatrics recomenda uma combinação de aspirina e IgIV para o tratamento de DK aguda. A IgIV tem sido usada com sucesso no tratamento da maioria dos casos de DK e tornou-se o padrão de tratamento. A melhor resposta é vista se o tratamento é iniciado dentro de 10 dias do início dos sintomas com uma dose única de $2 \mathrm{~g} / \mathrm{kg}$ com infusão durante 12 horas.

O mecanismo de ação proposto da IgIV inclui neutralização de antígenos infecciosos e superantígenos, supressão de TNF- $\alpha$, neutralização de autoanticorpos patogênicos, inibição de liberação de citocinas inflamatórias e modulação da função das células B e T (AGARWAL S e AGRAWAL DK, 2017).

\section{Correlação etiológica e imunológica entre Doença de Kawasaki e COVID-19:}

Em 1967, o Dr. Tomisacu Kawasaki publicou sua série detalhada de casos de 50 crianças, durante um surto de rinite, que desenvolveram uma condição que ele denominou como "Síndrome dos linfonodos mucocutâneos febris agudos" (LOKE YH, et al., 2020). 
A etiologia da DK ainda é desconhecida, porém, após o surto o Dr. Tomisacu Kawasaki começou a pensar em uma correlação entre a infecção viral e a doença que levou seu nome (CALABRI GB e FORMIGARI R, 2020). Além disso, o aumento de infiltrado plasmático de imunoglobulina $A(\lg A)$ e macrófagos no epitélio brônquico, inflamação dos tecidos de forma aguda, como nas artérias coronárias e nos linfonodos, presente na DK, é um padrão semelhante à resposta imune inata e adaptativa contra infecções virais.

Curiosamente, uma resposta vigorosa de $\lg A$ no hospedeiro foi detectada no estágio inicial da infecção pelo novo Coronavírus, o que pode sugerir uma possível ligação entre o novo Coronavírus e a DK. Desse modo, a presença de infiltrados inflamatórios e antígenos no trato respiratório e nos tecidos-alvo da DK pode indicar que o agente infeccioso apresenta tropismo pelos tecidos respiratório e vascular (RABA AA e ABOBAKER A, 2020).

Embora a relação entre a doença de Kawasaki e o novo Coronavírus ainda não esteja definida, há uma crescente preocupação com a síndrome inflamatória relacionada à infecção por SARS-CoV-2 como um possível elo entre a infecção por Coronavírus e a DK que afeta escolares (XU S, et al., 2020).

Nesse contexto, o dano epitelial por SARS-CoV-2 em estágio inicial, assim como na DK, pode induzir à endotelite local secundária, o que pode explicar um fenótipo vasculítico autoinflamatório tardio com regulação positiva de interleucinas (IL), como IL-1 $\beta$ ou IL-6 (POULETTY M, et al., 2020). Essa inflamação endotelial aumentada e lesão/disfunção após a infecção pelo novo Coronavírus, provavelmente, é devido à ação da enzima conversora de angiotensina (ECA) (XU S, et al., 2020).

A resposta inflamatória sistêmica à infecção pode potencializar a resposta inflamatória nas lesões coronárias, causando disfunção endotelial, acelerando o desenvolvimento da DK. Portanto, a infecção e a hiperinflamação no novo Coronavírus podem estar atuando como o "gatilho primário" que pode levar à DK (XU S, et al., 2020).

Ao contrário da DK clássica, a inflamação sistêmica após a infecção pelo novo Coronavírus afeta crianças e adolescentes. Outras descobertas intrigantes são que os pacientes com formas graves são menos caucasianos do que a frequência esperada em sua população em geral, sendo muitos da etnia afro-americana ou afro-caribenha. O envolvimento miocárdico é uma marca registrada desse estado hiperinflamatório, enquanto os aneurismas coronarianos são a marca registrada da DK (KONÉ-PAUT I e CIMAZ R, 2020).

Além de tudo, quase 6 semanas após o primeiro surto de novo Coronavírus na França, relatou-se uma série de 16 pacientes apresentando características de uma doença inflamatória sistêmica associada a uma infecção pelo novo Coronavírus denominada Kawa-COVID-19 (POULETTY M, et al., 2020). Ademais, alguns pacientes infectados pelo novo Coronavírus, devido à tempestade de citocinas, desenvolvem linfohistiocitose hemofagocítica secundária (sHLH) e Síndrome de Disfunção Respiratória Aguda (SDRA), enquanto outros, apesar de uma liberação consistente de citocinas pró-inflamatórias, não cumprem os critérios de sHLH, mas ainda mostram algumas características semelhantes ao fenótipo do Síndrome Hiperferritinêmica (ALUNNO A, et al., 2020).

Neste cenário, a linfohistiocitose hemofagocítica (HLH) é uma condição hiperinflamatória e hiperferritinêmica causada por células $T$, caracterizada por grande quantidade de interferon $Y$ (IFN-Y), estimulação dependente de receptores do tipo Toll (TLRs), células apresentadoras de antígenos e ativação descontrolada das células $T$, levando a uma tempestade de citocinas, caracterizando a doença inflamatória multissistêmica semelhante à síndrome do choque tóxico (SCT) e à doença de Kawasaki atípica (DK) (ALUNNO A, et al., 2020).

$\mathrm{Na}$ fase aguda da doença, pacientes com DK podem ter instabilidade hemodinâmica, uma condição conhecida como Síndrome de Choque da Doença de Kawasaki (SCDK), sendo que outros pacientes com DK podem preencher os critérios da Síndrome de Ativação de Macrófagos (MAS), semelhante à linfohistiocitose hemofagocítica secundária (sHLH) (VERDONI L, et al., 2020). A fisiopatologia da SCDK permanece incerta, porém um alto nível de citocinas pró-inflamatórias circulantes pode contribuir para o componente distributivo do choque. De fato, foi encontrada associada a altos níveis de interleucina-6 (IL-6), proteína C reativa e procalcitonina (TOUBIANA J, et al., 2020). 
Do ponto de vista imunológico, a gravidade da infecção pelo novo Coronavírus parece correlacionar-se com uma quantidade aumentada de várias citocinas, chamada tempestade de citocinas, em particular as interleucinas (IL), como IL-1 $\beta$, IL-2, IL-6, IL-7, IL-10, fator de necrose tumoral (TNF) $\alpha$, fator estimulador de colônias de granulócitos (G-CSF), interferon (IFN) $\mathrm{Y}$, proteína quimioatraente de monócitos, macrófagos e proteína inflamatória 1-a no soro e nos tecidos afetados. Dessa forma, as células T CD4+ são ativadas para se tornarem células Th1 patogênicas e gerar G-CSF, aumentando assim a expressão de IL-6 em monócitos.

Essa liberação maciça de mediadores pró-inflamatórios, alimentada por ciclos de feedback positivo e ativação aberrante do sistema imunológico, se assemelha à chamada síndrome de liberação de citocinas, um grupo de condições que compartilham o mesmo mecanismo patogênico, embora com uma diferente etiologia (ALUNNO A, et al., 2020).

Resumidamente, os Coronavírus suprimem as respostas imunes inatas para obter uma replicação eficiente. Nos casos mais graves, o hospedeiro apresenta uma resposta imune com uma reação excessiva retardada, incluindo uma tempestade de citocinas com uma alta quantidade de secreção de IL-6.

Os macrófagos podem ser as células mais responsáveis pela liberação dessas citocinas, uma vez que pacientes infectados geralmente apresentam linfopenia acentuada e abrigam uma população expandida de monócitos circulantes que secretam IL-6 e IL-1 $\beta$. No entanto, a resposta excessiva adaptativa das células T, células $B$ e até células epiteliais e endoteliais, poderia contribuir para essa tempestade de citocinas (BERTHELOT JM, 2020).

\section{Casos que demonstraram a relação entre Doença de Kawasaki e COVID-19}

O primeiro caso relatado de coinfecção de Doença de Kawasaki (DK) e COVID-19 foi publicado em 7 de abril de 2020, nos Estados Unidos, ocorrendo em um lactente de 6 meses de idade. Posteriormente, vários relatos de casos foram publicados em países como Reino Unido, Itália, Espanha, França, Suíça e na América Latina. A principal característica em comum entre os relatos publicados foi a presença de uma síndrome inflamatória multissistêmica grave que se assemelha à DK típica e incompleta, síndrome do choque tóxico (SCT) e síndrome de ativação macrofágica (MAS) (CAMPOS LR, et al., 2020).

Em Londres, Inglaterra, relataram-se características clínicas e laboratoriais de um grupo de 8 crianças com choque hiperinflamatório, todas com teste positivo para anticorpos SARS-CoV-2, sendo que as características clínicas desses casos compartilham características com síndrome de choque tóxico, Doença de Kawasaki e Síndrome de Choque da Doença de Kawasaki, incluindo febre, choque e erupção cutânea variada, conjuntivite, edema nas extremidades e sintomas gastrointestinais (CHIOTOS K, et al., 2020).

Em um estudo na Itália, Verdoni L, et al. (2020), dividiram todos os pacientes que foram diagnosticados com uma doença do tipo Kawasaki, nos últimos 5 anos, em dois grupos. O grupo 1 foram os pacientes com doença do tipo Kawasaki antes do início da pandemia do novo Coronavírus e o grupo 2 foram os pacientes com a mesma apresentação após a pandemia, descobrindo um aumento de 30 vezes na Doença de Kawasaki após a pandemia.

Eles também descobriram que casos mais graves foram encontrados após o surto pelo novo Coronavírus e os pacientes com DK durante esta pandemia eram mais velhos do que antes. Essas crianças apresentaram mais sinais de comprometimento cardíaco e ativação de macrófagos características da síndrome. Assim, notou-se que a pandemia está associada a casos graves de DK (ALIZARGAR J, 2020).

Também, Esper F, et al. (2005), observaram que entre onze pacientes consecutivos com doença de Kawasaki, oito tiveram swabs nasais positivos para o novo Coronavírus (CALABRI GB e FORMIGARI R, 2020). No Hospital Mount Sinai, em Nova York, destaca-se o relato de 15 possíveis casos de DK em pacientes pediátricos, a maioria diagnosticada com o novo Coronavírus. Possíveis casos de crianças com ambas as condições na Califórnia e Illinois também foram mencionados (SANDHAUS H, et al., 2020).

VERDONI L, et al. (2020), descreveram dez casos de doença do tipo Kawasaki que ocorre em Bergamo, Itália, no auge da pandemia no país, uma incidência mensal cerca de 30 vezes superior à observada na doença de Kawasaki nos últimos 5 anos (SARDU C, et al., 2020). 
Recentemente, 17 crianças com sinais e sintomas consistentes com a doença de Kawasaki e evidências laboratoriais de infecção recente pelo novo Coronavírus foram relatadas nos Estados Unidos $(n=1)$, Inglaterra $(n=8)$ e Itália $(n=8)$ (TOUBIANA J, et al., 2020).

\section{CONCLUSÃO}

Nos últimos 20 anos, os vírus da família dos Coronavírus foram propostos como participantes na patogênese da doença de Kawasaki (DK). O primeiro caso simultâneo de novo Coronavírus e DK foi a primeira descrição de uma criança com DK clássica, que também apresentou resultado positivo para COVID-19 em situações de febre e sintomas respiratórios mínimos. O vínculo definitivo entre a doença de Kawasaki (DK) e o novo Coronavírus precisa ser confirmado em uma grande coorte de bebês e crianças em todo o mundo, pois é importante para o atendimento pediátrico de crianças doentes, e as evidências disponíveis ainda são insuficientes. Assim, a associação documentada levanta a preocupação de saber se a infecção por COVID19 aumenta o risco de DK.

\section{REFERÊNCIAS}

1. AGARWAL S, AGRAWAL DK. Doença de Kawasaki: etiopatogenia e novas estratégias de tratamento. Revisão especializada de imunologia clínica, 2017; 13(3): 247-258.

2. ALIZARGAR J. The novel coronavirus (COVID-19) and the risk of Kawasaki disease in children. Journal of the Formosan Medical Association, 2020.

3. ALUNNO A, et al. Storm, typhoon, cyclone or hurricane in patients with COVID-19? Beware of the same storm that has a different origin. RMD Open, 2020; 6(1): e001295.

4. BERTHELOT JM. Kawasaki-like diseases and thrombotic coagulopathy in COVID-19: delayed over-activation of the STING pathway?. Emerging microbes \& infections, 2020; 1-26.

5. CALABRI GB, FORMIGARI R. Covid-19 and Kawasaki Disease: A Glimpse at the Past for a Predictable Future. Pediatric Cardiology, 2020.

6. CAMPOS LR, et al. Pediatric inflammatory multisystem syndrome (PIMS) temporally related to SARS-CoV-2. RP, 2020.

7. CHIOTOS K, et al. Multisystem Inflammatory Syndrome in Children during the COVID-19 pandemic: a case series. Journal of the Pediatric Infectious Diseases Society, 2020.

8. DEY M, ZHAO SS. COVID-19 and Kawasaki disease: an analysis using Google Trends. Clinical Rheumatology, 2020;1-2.

9. DEZA LEON MP, et al. COVID-19-associated pediatric multisystem inflammatory syndrome. Journal of the Pediatric Infectious Diseases Society, 2020; 9(3): 407-408.

10. DURSUN R, TEMIZ SA. The Clinics of HHV-6 infection in COVID-19 pandemic: Pityriasis rosea and Kawasaki disease. Dermatologic Therapy, 2020; e13730.

11. GARG S. Hospitalization rates and characteristics of patients hospitalized with laboratory-confirmed coronavirus disease 2019. Morbidity and mortality weekly report, 2020; 69: s/p.

12. GONÇALVES LF, et al. Kawasaki disease and COVID-19 in Children: A Systematic Review. SCIELO Preprints, 2020.

13. GRIMAUD $M$, et al. Acute myocarditis and multisystem inflammatory emerging disease following SARS-CoV-2 infection in critically ill children. Annals of Intensive Care, 2020; 10(1): 1-5.

14. HARAHSHEH AS, et al. Missed or delayed diagnosis of Kawasaki disease during the 2019 novel coronavirus disease (COVID-19) pandemic. The Journal of pediatrics, 2020.

15. JONES VG, et al. COVID-19 and Kawasaki disease: novel virus and novel case. Hospital Pediatrics, 2020; 10(6): 537540.

16. KAM KQ, et al. Kawasaki disease in the COVID-19 era: a distinct clinical phenotype?. The Lancet Child \& Adolescent Health, 2020.

17. KATO H, et al. Clinical course of 2019 novel coronavirus disease (COVID-19) in individuals present during the outbreak on the Diamond Princess cruise ship. Journal of Infection and Chemotherapy, 2020.

18. KONÉ-PAUT I, CIMAZ R. Is it Kawasaki shock syndrome, Kawasaki-like disease or pediatric inflammatory multisystem disease? The importance of semantic in the era of COVID-19 pandemic. RMD open, 2020; 6(2): e001333.

19. LABÉ P, et al. Erythema multiforme and Kawasaki disease associated with COVID-19 infection in children. Journal of the European Academy of Dermatology and Venereology, 2020.

20. LOKE YH, et al. Multisystem Inflammatory Syndrome in Children: Is There a Linkage to Kawasaki Disease?.Trends in Cardiovascular Medicine, 2020.

21. LOOMBA RS, et al. Covid-19 and Kawasaki syndrome: should we really be surprised?. Cardiology in the Young, 2020; 1-5.

22. MCCRINDLE BW, MANLHIOT C. SARS-CoV-2-Related Inflammatory Multisystem Syndrome in Children: Different or Shared Etiology and Pathophysiology as Kawasaki Disease?. JAMA, 2020. 
23. MOREIRA A. Kawasaki disease linked to COVID-19 in children. Nature Reviews Immunology, 2020.

24. POULETTY $M$, et al. Paediatric multisystem inflammatory syndrome temporally associated with SARS-CoV-2 mimicking Kawasaki disease (Kawa-COVID-19): a multicentre cohort. Annals of the Rheumatic Diseases, 2020.

25. PRUC M, et al. Kawasaki disease shock syndrome or toxic shock syndrome in children and the relationship with COVID-19. Medical Hypotheses, 2020; e109986.

26. RABA AA, ABOBAKER A. Covid-19 and Kawasaki Disease: An Etiology or Coincidental Infection?. The Pediatric Infectious Disease Journal, 2020.

27. RAUF A, et al. Multisystem inflammatory syndrome with features of atypical Kawasaki disease during COVID-19 pandemic. The Indian Journal of Pediatrics, 2020; 1-3.

28. RIGANTE D. Kawasaki Disease as the Immune-Mediated Echo of a Viral Infection. Mediterranean Journal of Hematology and Infectious Diseases, 2020; 12(1): s/p.

29. RIVERA-FIGUEROA El, et al. Incomplete Kawasaki Disease in a Child with Covid-19. Indian pediatrics, 2020.

30. ROWLEY AH, SHULMAN ST. The Epidemiology and Pathogenesis of Kawasaki Disease. Frontiers in Pediatrics, 2018.

31. SANDHAUS $\mathrm{H}$, et al. Association Between COVID-19 and Kawasaki Disease: Vigilance Required From Otolaryngologists. Otolaryngology-Head and Neck Surgery, 2020; e0194599820930238.

32. SARDU C, et al. Hypertension, thrombosis, kidney failure, and diabetes: Is COVID-19 an endothelial disease? A comprehensive evaluation of clinical and basic evidence. Journal of clinical medicine, 2020; 9(5): 1417.

33. SCHROEDER AR, et al. COVID-19 and Kawasaki disease: finding the signal in the noise. Hospital Pediatrics, 2020.

34. SOKOLOVSKY S, et al. COVID-19 associated Kawasaki-like multisystem inflammatory disease in an adult. The American Journal of Emergency Medicine, 2020.

35. TOUBIANA J, et al. Kawasaki-like multisystem inflammatory syndrome in children during the covid-19 pandemic in Paris, France: prospective observational study. BMJ, 2020; 369: s/p.

36. VERDONI L, et al. An outbreak of severe Kawasaki-like disease at the Italian epicentre of the SARS-CoV-2 epidemic: an observational cohort study. The Lancet, 2020.

37. VINER RM, WHITTAKER E. Kawasaki-like disease: emerging complication during the COVID-19 pandemic. The Lancet, 2020; 395(10239): 1741-1743.

38. WALTUCH T, et al. Features of COVID-19 post-infectious cytokine release syndrome in children presenting to the emergency department. The American Journal of Emergency Medicine, 2020.

39. XU S, et al. COVID-19 and Kawasaki disease in children. Pharmacological Research, 2020; 159: 104951. 